\title{
Asunaprevir Evokes Hepatocytes Innate Immunity to Restrict the Replication of Hepatitis C and Dengue Virus
}

\section{Citation}

Tsai, Wei-Lun, Jin-Shiung Cheng, Chih-Wen Shu, Kwok-Hung Lai, Hoi-Hung Chan, ChunChing Wu, Jing-Mei Wu, Ping-I Hsu, Raymond T. Chung, and Tsung-Hsien Chang. 2017. "Asunaprevir Evokes Hepatocytes Innate Immunity to Restrict the Replication of Hepatitis C and Dengue Virus." Frontiers in Microbiology 8 (1): 668. doi:10.3389/fmicb.2017.00668. http:// dx.doi.org/10.3389/fmicb.2017.00668.

\section{Published Version}

doi:10.3389/fmicb.2017.00668

\section{Permanent link}

http://nrs.harvard.edu/urn-3:HUL.InstRepos:33029729

\section{Terms of Use}

This article was downloaded from Harvard University's DASH repository, and is made available under the terms and conditions applicable to Other Posted Material, as set forth at http:// nrs.harvard.edu/urn-3:HUL.InstRepos:dash.current.terms-of-use\#LAA

\section{Share Your Story}

The Harvard community has made this article openly available.

Please share how this access benefits you. Submit a story.

Accessibility 


\section{OPEN ACCESS}

Edited by:

Shiu-Wan Chan,

University of Manchester, UK

Reviewed by:

Mathias Faure,

Claude Bernard University Lyon 1 .

France

Youichi Suzuki,

Osaka Medical College, Japan

*Correspondence:

Wei-Lun Tsai

tsaiw@yahoo.com.tw

Tsung-Hsien Chang

changth@vghks.gov.tw

${ }^{\dagger}$ These authors have contributed equally to this work.

Specialty section: This article was submitted to

Virology,

a section of the journal

Frontiers in Microbiology

Received: 15 October 2016

Accepted: 31 March 2017

Published: 20 April 2017

Citation:

Tsai W-L, Cheng J-S, Shu C-W, Lai K-H, Chan H-H, Wu C-C, WU J-M, Hsu P-I, Chung RT and Chang T-H

(2017) Asunaprevir Evokes

Hepatocytes Innate Immunity to Restrict the Replication of Hepatitis C and Dengue Virus.

Front. Microbiol. 8:668.

doi: 10.3389/fmicb.2017.00668

\section{Asunaprevir Evokes Hepatocytes Innate Immunity to Restrict the Replication of Hepatitis C and Dengue Virus}

\author{
Wei-Lun Tsai ${ }^{1,2 *}$, Jin-Shiung Cheng ${ }^{1,2 \dagger}$, Chih-Wen Shu ${ }^{3}$, Kwok-Hung Lai ${ }^{1,2}$, \\ Hoi-Hung Chan ${ }^{1,2}$, Chun-Ching Wu ${ }^{1}$, Jing-Mei Wu ${ }^{1}$, Ping-I Hsu ${ }^{1,2}$, Raymond T. Chung ${ }^{4}$ \\ and Tsung-Hsien Chang ${ }^{3,5 *}$ \\ 1 Division of Gastroenterology and Hepatology, Department of Internal Medicine, Kaohsiung Veterans General Hospital, \\ Kaohsiung, Taiwan, ${ }^{2}$ School of Medicine, National Yang-Ming University, Taipei, Taiwan, ${ }^{3}$ Gastrointestinal Unit, Department \\ of Medicine, Massachusetts General Hospital, Harvard University, Boston, MA, USA, ${ }^{4}$ Department of Medical Education and \\ Research, Kaohsiung Veterans General Hospital, Kaohsiung, Taiwan, ${ }^{5}$ Department of Medical Laboratory Science and \\ Biotechnology, Chung Hwa University of Medical Technology, Tainan, Taiwan
}

Type I Interferon-mediated innate immunity against Flaviviridae, such as Hepatitis C virus (HCV) and Dengue virus (DENV), involves TLR3, RIG-I-like receptor (RLR) and JAK-STAT signal pathways. Asunaprevir is a newly developed HCV protease inhibitor for HCV treatment. Whether, asunaprevir activates innate immunity to restrict viral infection is unclear. Thus, this study investigates the effect of asunaprevir on innate immunity and its influence on HCV and DENV infection. Huh 7.5.1, Hep-G2 cells, JFH-1 infection model, and DENV-2 infection were used for the analysis. The activity of asunaprevir-regulated innate immunity signal pathway was assessed with IFN- $\beta$ promoter or IFN-stimulated responsive element (ISRE) reporter assays and immunoblotting of key signal proteins. siRNA-mediated MAVS and TRIF knockdown of cells was performed to assess the effect of asunaprevir-regulated innate immunity against HCV and DENV. Asunaprevir treatment activated ISRE and IFN- $\beta$ promoter-luciferase activities and signaling proteins in the JAK-STAT, MAVS, and TRIF pathways in Huh 7.5.1 cells. Asunaprevir-mediated signaling activation was decreased in MAVS-knockdown cells. Importantly, both RNA and protein levels of DENV-2 NS3 were decreased in asunaprevir-treated Huh 7.5.1 and HepG2 cells. In MAVS-knockdown cells, the restrictive effect of asunaprevir on HCV and DENV was attenuated. Our findings reveal an unexpected activity of asunaprevir, the activation of MAVS dependent innate immunity to restrict HCV and DENV infection.

Keywords: hepatitis C, Dengue virus, innate immunity, interferon type I, asunaprevir

\section{INTRODUCTION}

Currently, as much as $4 \%$ of the world's population is chronically infected with hepatitis $\mathrm{C}$ virus (HCV; Kao and Chen, 2005). After 20-30 years of infection, 20-30\% of chronic HCV patients will develop cirrhosis of liver or hepatocellular carcinoma (Fattovich et al., 2004). An estimated 2.5 billion people are living in areas where dengue is epidemic, leading to 50-100 million human infections each year (WHO, 2009, 2012). Dengue virus (DENV) infection frequently leads to 
dengue fever, life-threatening dengue hemorrhagic fever (DHF), or dengue shock syndrome (DSS; Gubler, 1989; WHO, 2009, 2012). Despite the tremendous efforts in anti-Dengue viral research, no clinically approved antiviral agents are available and current treatment of DENV infection is only supportive care (Vaughn et al., 2000; Noble et al., 2010).

Resolution of the three-dimensional structures of several HCV proteins, together with the development of replicative cell culture systems, has led to the identification of a number of potential targets for direct-acting antiviral (DAA) agents (Ploss and Dubuisson, 2012; Schaefer and Chung, 2012). It has been confirmed that IFN-free regimens can lead to HCV eradication (Liang and Ghany, 2014). Recent research has shown that dual therapy with asunaprevir, a NS3 inhibitor, and daclatasvir, an NS5A inhibitor, in HCV genotype 1b-infected null responders resulted in an nearly $90 \%$ SVR rates (Chayama et al., 2012). In another study, treatment of asunaprevir and dalactasvir resulted in an SVR rate of $90 \%$ in genotype $1 \mathrm{~b}$ treatment naive patients (Manns et al., 2014). IFN $\alpha$ can eradicate $\mathrm{HCV}$ through activation of IFN signaling pathway and activation of IFN-stimulated genes (ISGs; Sameul, 2001; Tai and Chung, 2009; Bartenschlager et al., 2012). Whether DAAs exert immune regulatory effects in the treatment of HCV remains unclear.

Innate immunity to HCV and DENV is triggered by activation of cellular sensors that recognize the presence of pathogenassociated molecular patterns (PAMPs). The two key cellular sensors are Toll-like receptor 3 (TLR3), which senses doublestranded RNA (dsRNA) within endosomes, and the RNA helicase retinoic acid-inducible gene I (RIG-I), which senses intracellular double-stranded RNA or single-stranded viral RNA (Wang et al., 2009; Green et al., 2012, 2014; Li et al., 2012; Horner and Gale, 2013). Signaling initiated by TLR3 and RIG-I is transmitted through the adaptor proteins Toll/IL-1 receptor domain-containing adaptor inducing IFN-beta (TRIF) and mitochondrial antiviral-signaling protein (MAVS) respectively, to interact with TNF receptor-associated factor 3 (TRAF3) and converge on the transcription factors, interferon regulatory factor 3 (IRF3) and NF- $\kappa$ B. IRF3 is phosphorylated by IKK- $\varepsilon$ and TANK-binding kinase 1 (TBK1), resulting in its dimerization and nuclear translocation.

Activated IRF3 and NF- $\kappa \mathrm{B}$ translocate to the nucleus, where they activate IFN- $\beta$ gene transcription. The secreted type I IFN cytokine stimulates the IFN- $\alpha / \beta$ receptor (IFNAR-1 and -2) through autocrine or paracrine interaction and then activates the Janus kinase-signal transducer and activator of transcription (JAK-STAT) signaling pathway (Schindler and Plumlee, 2008; Schoggins et al., 2011). The STAT proteins become phosphorylated by the JAK family members JAK-1 and TYK2. Phosphorylated STAT-1 and STAT-2 recruit IRF9 to form a complex known as IFN-stimulated gene factor 3 (ISGF3), which translocates into the nucleus, binds to IFN-stimulated response elements (ISRE) and induces the transcription of ISGs, many of which are thought to confer antiviral effects including HCV and Dengue virus (Sameul, 2001; Schindler and Plumlee, 2008; Schoggins and Rice, 2011; Schoggins et al., 2011; Fusco et al., 2013).
$\mathrm{HCV}$ directs a variety of viral strategies to disrupt host innate immune defenses through its viral proteins (Bartenschlager et al., 2012; Horner and Gale, 2013). The HCV NS3-4A protease specifically cleaves both MAVS and TRIF to inactivate signals initiated by RIG-I and TLR3 for anatomization of innate immunity. We therefore hypothesized that a distinct HCV protease inhibitor can activate innate immunity. This study first investigated the effects of asunaprevir on hepatoma cells, and found interestingly that asunaprevir activates innate immunity in a MAVS dependent manner in hepatoma cells. In view of these findings, the impact of asunaprevir in activation of innate immunity against HCV and Dengue virus infection was examined.

\section{MATERIALS AND METHODS}

\section{Cells, Virus, and Reagents}

Huh 7.5.1 cells were grown in Dulbecco's Modified Eagle's Medium (DMEM) supplemented with $10 \%$ fetal bovine serum (FBS). The infectious JFH1 plasmid was obtained from Dr. Takaji Wakita and inoculated as previously described (Lin et al., 2010). DENV-2 PL046 (Genbank accession \# AJ968413.1) isolated from patients with dengue fever was kindly provided by Lin et al. (1998). These viruses were propagated in mosquito cell line C6/36 (ATCC: CRL-1660) grown in RPMI 1640 medium containing 5\% FBS. HepG2 cells were purchased from Bioresource Collection and Research Center (BCRC) and grown in DMEM supplemented with 10\% FBS. Asunaprevir was obtained from the Bristol-Myers Squibb Company.

\section{Luciferase Assay}

To monitor IFN signaling directed by ISRE, plasmids pISREluc (500 ng/well) expressing firefly luciferase and pRL-TK (50 ng/well) expressing Renilla luciferase as an internal control were co-transfected in Huh 7.5.1 cells $\left(1 \times 10^{5}\right)$. To monitor IFN- $\beta$ promoter signaling, pGL-4 IFN $\beta$-Luc (pIFN $\beta / F L u c) ~(500 \mathrm{ng} /$ well) expressing firefly luciferase and pRL-TK (50 ng/well) expressing Renilla luciferase as an internal control were used (Chang et al., 2006, 2009). Huh 7.5.1 cells were transfected with Lipofectamine 2000 Transfection Reagent (Invitrogen) following the manufacturer's protocol. Briefly, cells cultured in 12-well plates were transfected with a DNA precipitate containing plasmid DNA (amounts as indicated and had been adjusted by the vector control to be the same among samples of the experimental group). Five hours after transfection, cells were replenished with culture medium, and then incubated for various time points. Relative luciferase activity was assessed by the Promega dual-luciferase reporter assay system (Promega, Madison, WI).

\section{Immunofluorescence Assay}

Immunofluorescence staining of HCV core protein in Huh 7.5.1 cells treated with supernatant of JFH-1 infected Huh 7.5.1 cells and Immunofluorescence staining of DENV DV2NS3 protein in Huh 7.5.1 cells treated with supernatant of DENV infected Huh 7.5.1 cells were performed. Huh7.5.1 cells were fixed with $4 \%$ paraformaldehyde, permeabilized with $0.5 \%$ TritonX-100, and 
blocked with $3 \%$ bovine serum albumin in PBS. The primary antibody was mouse anti-HCV core (Thermoscientific) or antiDENV DV2NS3 (\#YH3304, Yao-Hong Biotechnology, Taipei). The secondary antibody was goat anti-mouse Alexa Fluor 488 (Invitrogen). DAPI was added to the staining to monitor the nuclear structure. Fluorescence signals were observed by fluorescence microscopy (Zeiss, Axcio Observer A1).

\section{Immunoblotting}

Cells were lysed using radioimmune precipitation assay (RIPA) buffer containing 1\% NP-40, 0.1\% SDS, $10 \mathrm{mM}$ Tris- $\mathrm{HCl}(\mathrm{pH}$ 7.4), $1 \mathrm{mM}$ EDTA, $150 \mathrm{mM} \mathrm{NaCl}$ and protease inhibitor cocktail (Roche), followed by sonification. Proteins were separated by SDS-PAGE with NuPAGE Novex pre-cast $4-12 \%$ Bis-Tris gradient gels (Invitrogen, Carlsbad, CA) and transferred to PVDF membranes. The primary antibodies used were anti-STAT1, antiphospho-STAT1, anti-STAT2, anti-phospho-STAT2, anti-MAVS, anti-TRIF, anti-PKR, anti-IRF3 (Cell Signaling Technology, Inc., Beverly, MA), anti-HCV core, (Thermoscientific), anti-HCV NS3, anti-HCV NS5A and anti-NS5B (Virogen, Watertown, MA), anti-ISG15, anti-MxA and anti-TRIF (Abcam), antiTRF3 (Santa Cruz), anti-phospho-IRF3 (Origene) and anti- $\beta$ actin (Sigma Life Science and Biochemicals, St. Louis, MO). DENV-2 NS3 was detected by a specific monoclonal antibody against NS3 (Yao-Hong Biotechnology). Secondary antibodies were HRP-conjugated ECL donkey anti-rabbit IgG and HRPconjugated ECL sheep anti-mouse IgG (Amersham Biosciences, Piscataway, NJ). The ECL Western Blotting Detection Kit (Amersham Biosciences, Piscataway, NJ) was employed to detect chemiluminescent signals. Immunoblots shown in each figure are representative of three independent experiments. Densitometry was performed with ImageJ software. Student's $t$-test was used as statistical test.

\section{Real-Time Quantitative PCR (qPCR)}

Total cellular and viral RNA was isolated post-infection using RNeasy Mini columns (QIAGEN) or Trizol reagent (Invitrogen) and reverse transcribed by random priming with the High Capacity cDNA Reverse Transcription Kit (Applied Biosystems; Foster City, CA). qPCR amplification involved $3 \mathrm{ng}$ cDNA in $10 \mu \mathrm{l}$ SYBR Green PCR master mix (Applied Biosystems) with $3 \mu \mathrm{M}$ primers in ABI StepONE Plus Real-Time PCR system (Applied Biosystems). Transcript levels were normalized to that of hypoxanthine phosphoribosyltransferase (HPRT). The primers used in this study are listed in Table 1.

TABLE 1 | List of primer sequences used in quantitative PCR.

\begin{tabular}{lll}
\hline Target gene & Primer & Nucleotide sequence \\
\hline GAPDH & Forward & 5'-CAACTGGTCGTGGACAACCAT-3' \\
& Reverse & 5'-GCACGGACACTCACAATGTTC-3' \\
JFH1 & Forward & 5'-TCTGCGGAACCGGTGAGTA-3' \\
& Reverse & 5'-TCAGGCAGTACCACAAGGC-3' \\
Dengue virus & Forward & 5-AGTTGTAGTCTACGTGGACCGA-3 \\
& Reverse & 5'-CGCGTTCAGCATATTGAAAG-3'
\end{tabular}

\section{siRNA and Transfection}

SMART pool siRNA for MAVS and TRIF for gene knockdown were purchased from Dharmacon. The negative control siRNA was obtained from QIAGEN. Lipofectamine ${ }^{\mathrm{TM}}$ RNAiMAX Transfection Reagent (Invitrogen, Carlsbad, CA) was used for siRNA transfection. Protein expression of each knockdowned gene was confirmed by immunoblotting.

\section{RESULTS}

\section{Asunaprevir Activates ISRE Reporter Activity in Huh 7.5.1 Cells}

To understand whether asunaprevir activates cellular type-I IFN mediated antiviral machinery, an ISRE-luciferase reporter assay was conducted to assess the STAT1/STAT2 activity of IFN pathway axis. Both pISRE/luc and pRL-TK (transfection control vector) were co-transfected into Huh 7.5.1 cells for $48 \mathrm{~h}$, and then treated with 1 or $10 \mathrm{nM}$ asunaprevir. The luciferase activity was then measured at 3,6, 24, and $48 \mathrm{~h}$ post-treatment. The data showed that Asunaprevir s-stimulated ISRE activity at 24 and $48 \mathrm{~h}$ post-treatment in Huh 7.5.1 cells with a dose-dependent manner (Figure 1A).

\section{Asunaprevir Activates Type I IFN Antiviral Signaling Pathway}

To prove that type I IFN-mediated antiviral signaling is regulated by asunaprevir, the signaling proteins in Huh 7.5.1 cells treated with different doses of Asunaprevir for $48 \mathrm{~h}$ were determined by immunoblotting analysis. Results showed that asunaprevir increased STAT1 and STAT2 expression and their phosphorylation in Huh 7.5.1 cells (Figure 1B, upper panels). Downstream ISGs such as MxA and ISG-15 were also induced in asunaprevir-treated cells, demonstrating that asunaprevir activated the JAK-STAT signaling pathway (Figure 1B, upper panels). The protein expression level was also analyzed by densitometry, which showed the consistent results (Figure 1B, lower panels).

\section{Asunaprevir Induces IFN- $\beta$ Reporter Activity in Huh 7.5.1 Cells}

To determine whether asunaprevir induces IFN- $\beta$ promoter activation with IFN- $\beta$ promoter luciferase reporter assay, the vectors of $\mathrm{pIFN}-\beta /$ Fluc and $\mathrm{pRL}-\mathrm{TK}$ were transfected into Huh 7.5.1 cells for $48 \mathrm{~h}$, and then treated with Asunaprevir (1, 10, and $100 \mathrm{nM})$. After $24 \mathrm{~h}$ stimulation, dual luciferase analysis was conducted. Results indicated activation of IFN$\beta$ promoter luciferase activity by asunaprevir in Huh 7.5.1 cells (Figure 1C). RNA virus infection-stimulated type I IFN production is dependent on the activation of TLR3 or RIG-I signaling pathway. Because Huh7.5.1 cells are RIG-1 deficient, therefore, this data suggested an unexpected role of asunaprevir in the activation of MAVS- mediated IFN- $\beta$ production signaling pathway. 
A

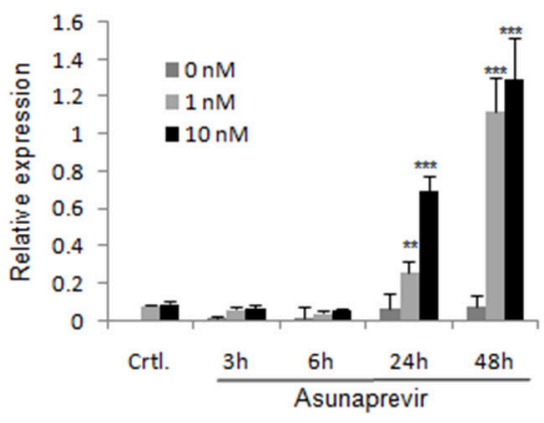

B
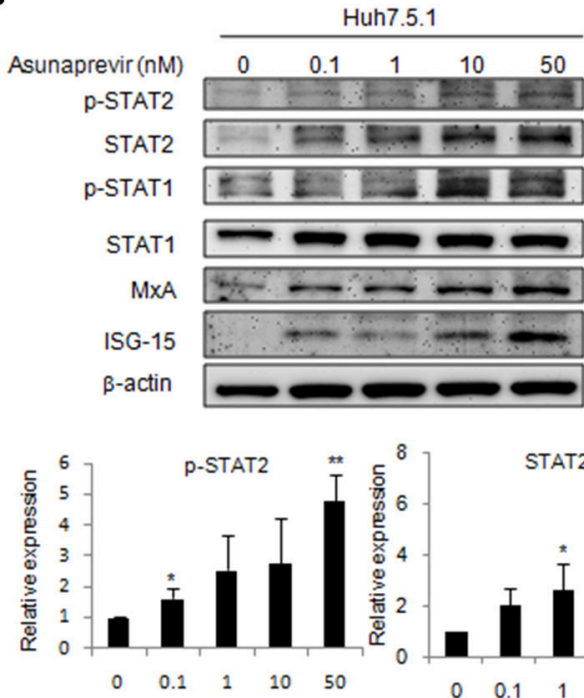

STAT2
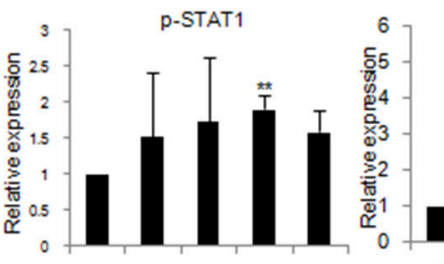

0.1

STAT1

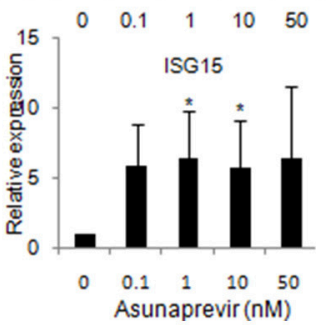

C

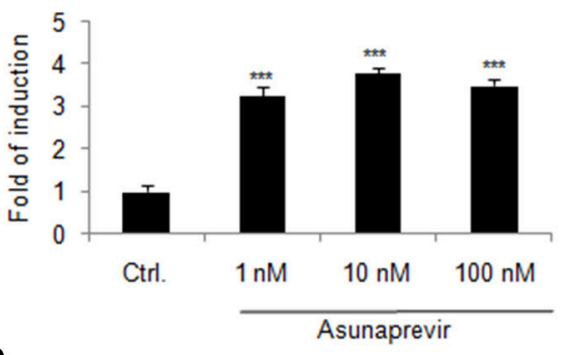

D Huh7.5.1
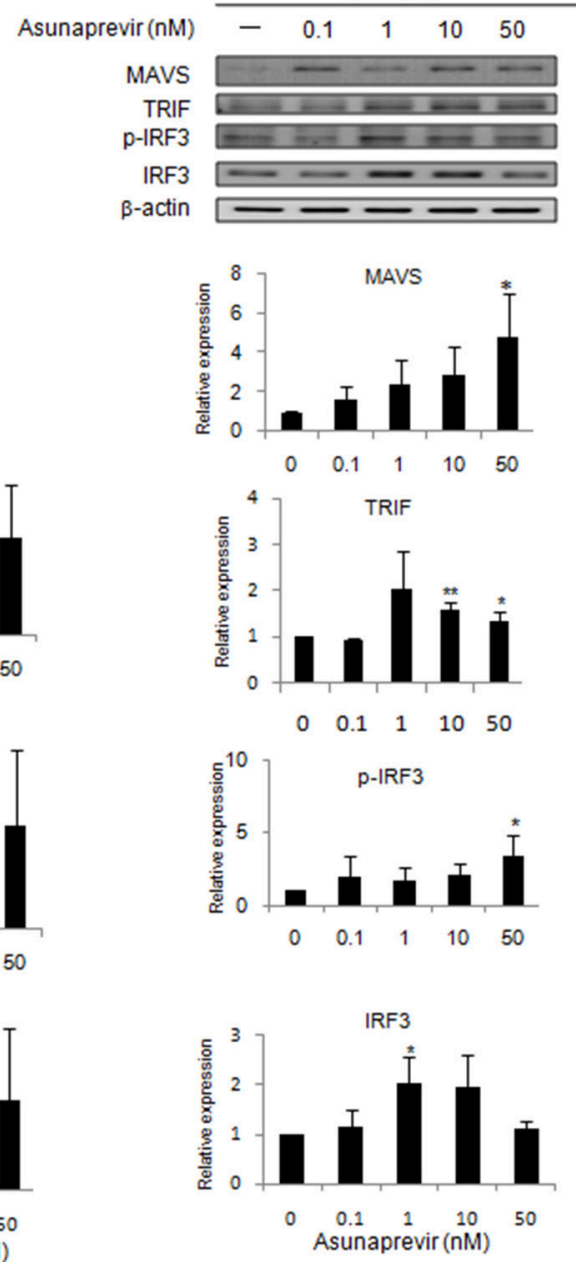

FIGURE 1 | Asunaprevir activates ISRE activity and type I IFN and TLR3/RIG-I antiviral signaling pathway. (A) ISRE luciferase reporter assay, plasmids of pISRE-luc expressing firefly luciferase and pRL-TK expressing Renilla luciferase as an internal control were co-transfected to Huh 7.5.1 cells and then treated with 1 or $10 \mathrm{nM}$ of asunaprevir for $3,6,24$, and $48 \mathrm{~h}$. The $0 \mathrm{nM}$ indicated DMSO vehicle control. The firefly and Renilla luciferase activities were then measured by dual-luciferase assay. Relative firefly luciferase activity was normalized to Renilla luciferase activity. (B) Huh 7.5.1 were treated with different doses of asunaprevir for 48 $\mathrm{h}$ and the cell lysates were analyzed by immunoblotting with the indicated antibodies involved in the interferon signaling pathway (upper panels). The protein levels of STAT-1, phosphorylated STAT-1, STAT-2, phosphorylated STAT-2, MxA, and ISG-15 relative to the $\beta$-actin were determined by densitometry with ImageJ software (lower, panels). (C) IFN- $\beta$ luciferase reporter assay, plasmids pIFN- $\beta$ /Fluc expressing firefly luciferase and pRL-TK expressing Renilla luciferase as an internal control were co-transfected to Huh 7.5.1 cells and then treated with 1, 10, or $100 \mathrm{nM}$ of asunaprevir for 24 h. Firefly and Renilla luciferase activities were then measured. Relative firefly luciferase activity was normalized to Renilla luciferase activity. (D) Huh 7.5.1 cells were treated with different doses of asunaprevir for $48 \mathrm{~h}$ and the cell lysates were analyzed by immunoblotting with the indicated antibodies involved in the TLR3/RIG-I signaling pathway (upper panels). The protein levels of MAVS, TRIF, IRF-3, and phosphorylated IRF-3 relative to the $\beta$-actin were shown at the bottom of each sample. Immunoblots shown in each figure are representative of three independent experiments. Densitometry was performed with ImageJ software (lower, panels). Values represent the average of three assays \pm S.D. Statistical significance was tested by Student's $t$-test, ${ }^{\star} P<0.05$, ${ }^{\star \star} P<0.01$, ${ }^{\star \star \star} P<0.001$. 


\section{Asunaprevir Activates the TLR3 or RIG-I Signaling Pathway}

To understand whether the TLR3 axis is regulated by asunaprevir, the signaling proteins TRIF, IRF-3, and phosphorylated IRF-3 in Huh 7.5.1 cells treated with different doses of asunaprevir for $48 \mathrm{~h}$ were determined by immunoblotting analysis, and the protein level was also analyzed by densitometry. Because the activation of MAVS independent of RIG-I was reported (Jacobs and Coyne, 2013), thus, the asunaprevir- induced MAVS was also determined. These results showed that asunaprevir increased MAVS and TRIF expression and also induced the phosphorylation of IRF-3 in Huh 7.5.1 cells (Figure 1D).

\section{MAVS Knockdown Abolishes Asunaprevir-Induced IRF3 Activation}

To validate the role of MAVS and TRIF in asunaprevirregulated innate immunity, siRNA-mediated knockdown of MAVS and TRIF expression was conducted in Huh 7.5.1 cells. Immunoblotting showed that asunaprevir-induced IRF3 phosphorylation was attenuated in Huh 7.5.1 cells after MAVS knockdown (Figure 2). However, TRIF knockdown was not able to reduce the level of asunaprevir-induced IRF3 phosphorylation. The results indicated that asunaprevir activates innate immune signaling pathway in a MAVS dependent manner.

\section{Asunaprevir Impairs DENV and HCV Replication}

As asunaprevir was found to activate host antiviral machinery, the effect of asunaprevir on DENV replication in Huh 7.5.1 and HepG2 cells was assessed. Huh 7.5.1 and HepG2 cells were infected with DENV and treated with asunaprevir for $24 \mathrm{~h}$, followed by immunoblotting analysis for NS3 protein of DENV and real-time PCR for RNA level of DENV-2. After treatment with asunaprevir, the protein level of NS3 and the RNA level of DENV-2 were decreased in Huh 7.5.1 (Figures 3A,B) and HepG2 cells (Figures 3C,D), suggesting that asunaprevir inhibited the replication of DENV in Huh 7.5.1 and HepG2 cells. Asunaprevir is known as a HCV inhibitor. Thus, the anti-HCV effect of Asunaprevir was also determined in our infection model. JFH1 infected Huh 7.5.1 cells were treated with asunaprevir for $24 \mathrm{~h}$, followed by real-time PCR for RNA level of HCV. The RNA level of HCV was decreased in JFH-1 infected Huh 7.5.1 (Figure 3E).

Moreover, the Asunaprevir restricted DENV-2 and HCV virus production was demonstrated. The DENV-2 and HCV production was determined using the supernatant from the DENV or JFH-1-infected Huh 7.5.1 cells with Asunaprevir treatment. The supernatant was harvested to infect Huh 7.5.1 cells, after $48 \mathrm{~h}$ post infection, the immunofluorescence assay of DENV-NS3 and HCV core proteins was conducted (Figure 4A). The data showed that asunaprevir inhibited the DENV-2 and $\mathrm{HCV}$ virus production (Figures $4 \mathrm{~B}, \mathrm{C}$ ).

\section{MAVS Activity Is Involved in the Anti-HCV Effect of Asunaprevir}

Asunaprevir-inhibited HCV replication is rescued in MAVS knockdowned cells. To understand the effect of asunapreviractivated innate immunity against HCV, both MAVS and TRIF were knocked down in JFH-1-infected Huh 7.5.1 cells, followed by treatment with asunaprevir. HCV core protein, MAVS and TRIF were determined by immunoblotting analysis and the protein expression level was determined by densitometry. After knockdown of MAVS with siRNA, asunaprevir-decreased HCV core protein level was partly rescued in JFH-1-infected Huh 7.5.1 cells (Figure 5). However, the HCV core protein recovery was not shown in TRIF knockdown cells. The data demonstrated that, except directly NS3 inhibition, asunaprevir also inhibit the replication of $\mathrm{HCV}$ in JFH-1 infected cells through the activation of MAVS mediated innate immune response additionally.
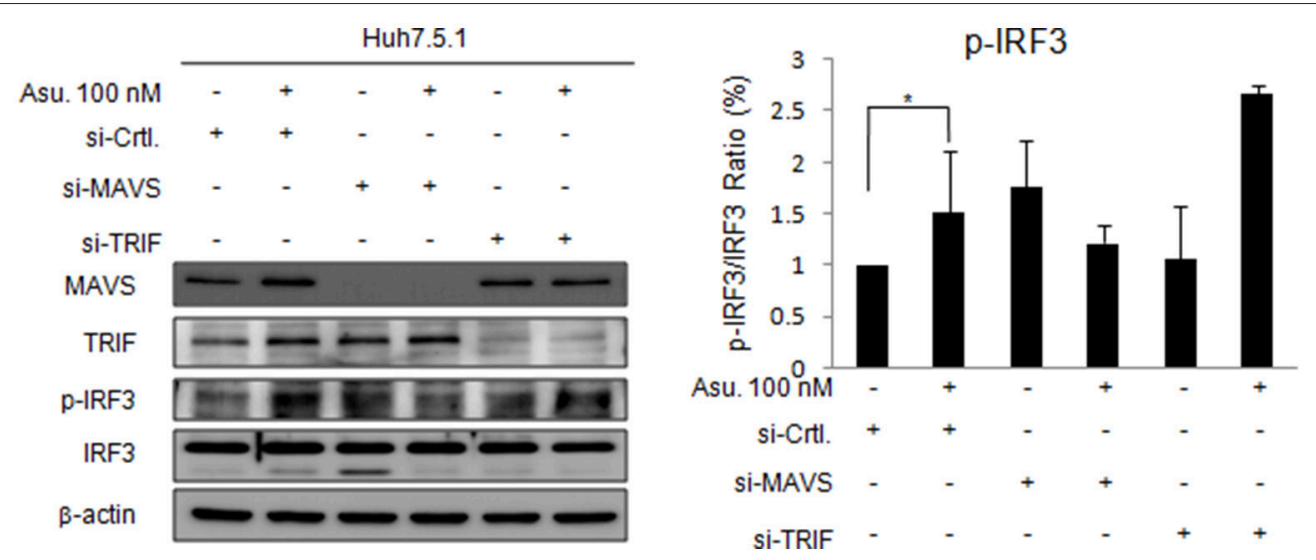

FIGURE 2 | Effects of Asunaprevir on TLR3/RIG-I signaling pathway in Huh. 7.5.1 cells with MAVS and TRIF knockdown. Huh 7.5.1 cells were transfected by siRNA of MAVS and TRIF for $48 \mathrm{~h}$ and then treated with asunaprevir for $24 \mathrm{~h}$. The key signaling proteins such as MAVS, TRIF, IRF3, and phosphorylated IRF-3 were determined by immunoblotting analysis (left panel). Immunoblots shown in the figure are representative of three independent experiments. The protein levels phosphorylated IRF-3 over total IRF3 was analyzed with ImageJ software (right panel). Data are mean $\pm S D$ from 3 independent tests. Statistical significance was tested by Student's $t$-test, ${ }^{*} P<0.05$. 
A
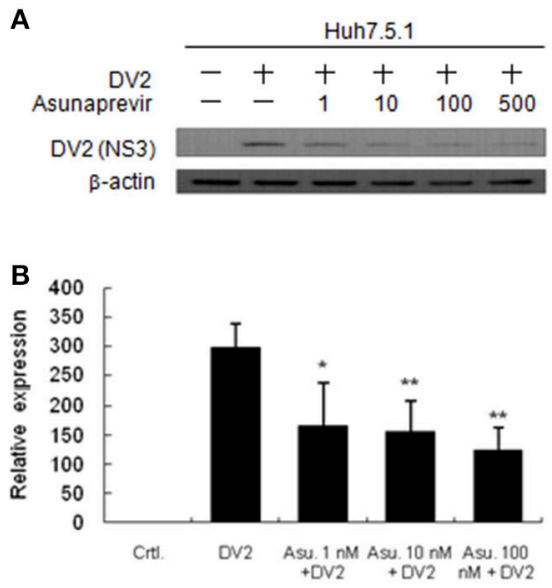

E

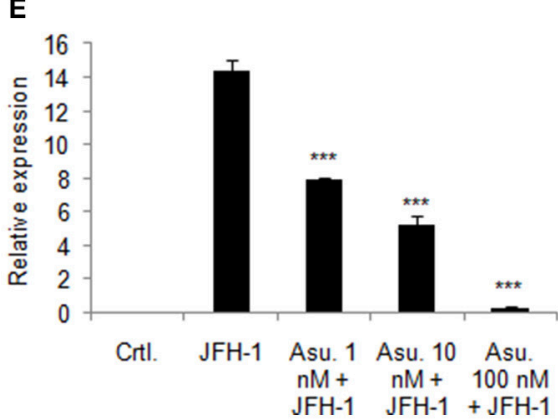

C

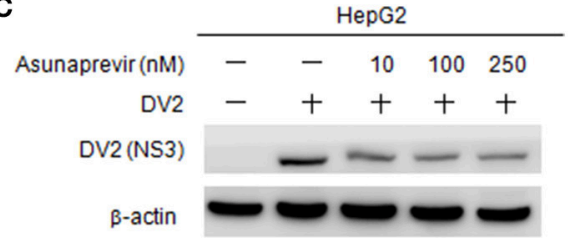

D

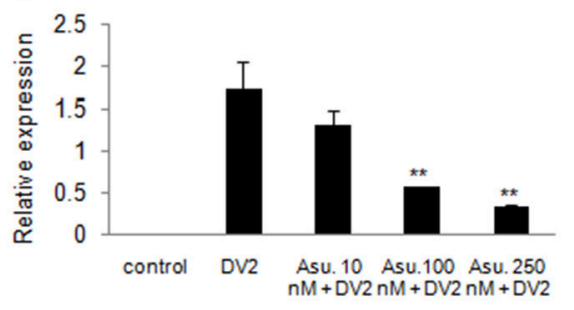

FIGURE 3 | Effects of Asunaprevir on replication of DENV and HCV. (A) Huh 7.5.1 cells were treated with different doses of asunaprevir for 24 h and immunoblotting analysis for NS3 protein of DENV, and real-time PCR for RNA level of DENV were performed (B). (C) HepG2 cells were treated with different doses of asunaprevir for $24 \mathrm{~h}$ and immunoblotting analysis for NS3 protein of DENV, and real-time PCR for RNA level of DENV were performed (D). (E) JFH-1 infected Huh 7.5.1 cells were treated with asunaprevir for $24 \mathrm{~h}$, followed by real-time PCR for RNA level of HCV. Data are mean $\pm S D$ from 3 independent tests. Statistical significance was tested by Student's $t$-test, ${ }^{\star} P<0.05,{ }^{* \star} P<0.01,{ }^{\star \star *} P<0.001$.

\section{Effect of Asunaprevir on Replication of DENV in Huh 7.5.1 Cells with MAVS and TRIF Knockdown}

To understand whether MAVS- and TRIF-mediated innate responses play roles in asunaprevir inhibition against DENV2 replication, Huh 7.5.1 cells infected with DENV-2 were transfected by siRNA of MAVS and TRIF for $48 \mathrm{~h}$ and then treated with asunaprevir. Immunoblotting analysis for NS3 protein of DENV, MAVS, and TRIF showed that asunaprevirdecreased DENV NS3 protein level was rescued in MAVS knockdowned Huh 7.5.1 cells (Figure 6A, upper panels). The densitometry analysis showed consistent results (Figure 6A, lower panel). These data demonstrated that asunaprevir inhibits the replication of DENV in Huh 7.5.1 cells through the activation of the MAVS mediated innate immune response.

\section{Effect of Asunaprevir on NS3 Protein of DENV in Huh 7.5.1 Cells}

Asunaprevir is a $\mathrm{HCV}$ protease inhibitor for HCV treatment. To verify whether asunaprevir inhibits DENV protease activity, the Huh7.5.1 cells were transfected with DNEV NS2B3 and treated with asunaprevir. The immunoblots showed the NS3 was processed from NS2B3 by its protease activity, which was not inhibited by asunaprevir (Figure 6B). The NS2B3 protease dead mutant (S135A) was used as the un-cleavage control. Our data confirmed that DENV2 protease was not targeted by asunaprevir. Taking together, our data suggest that asunaprevir elicits host antiviral activity against DENV and HCV beside to HCV protease inhibition.

\section{DISCUSSION}

This study found that asunaprevir activated innate immunity in hepatoma cells. Asunaprevir also restricted the replication of HCV and DENV through the activation of MAVS mediated innate immune response.

It has been recently confirmed that IFN-free regimens can lead to HCV eradication (Liang and Ghany, 2014). Daclatasvir and asunaprevir have received their first global approval in this indication in Japan and is the first all-oral, interferonand ribavirin-free regimen for this indication (Poole, 2014). In a recent study that investigated the effect of combined dalactasvir and asunaprevir treatment for chronic HCV, Manns et al. (2014) found that baseline dalactasvir, but not asunaprevir, 
A
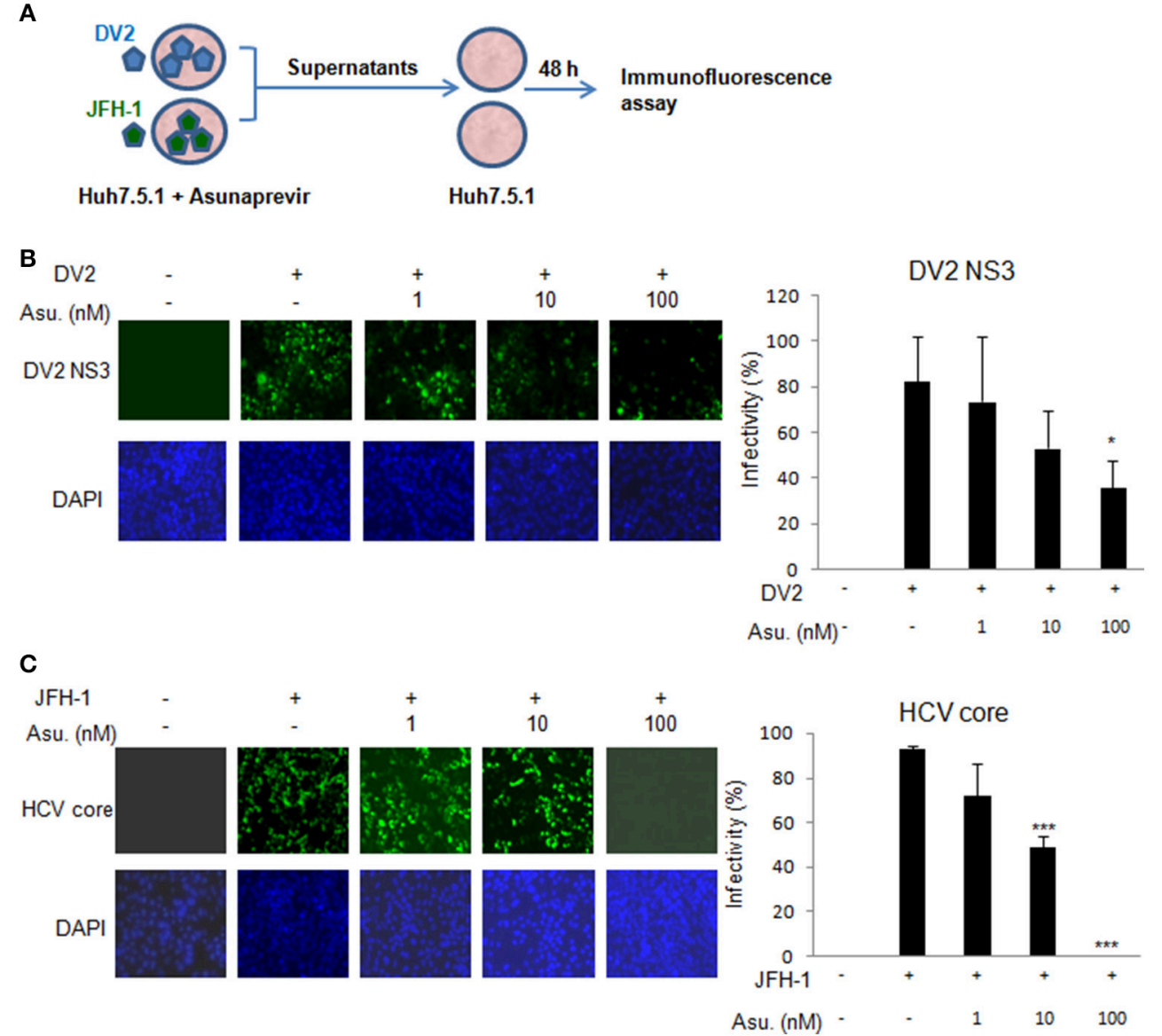

FIGURE 4 | Asunaprevir inhibits DENV-2 and HCV virus production. (A) The schematic of asunaprevir treatment in DENV and HCV infection in Huh 7.5.1 cells. The asunaprevir-treated Huh 7.5.1 cells were infected by DENV and JFH-1 for $48 \mathrm{~h}$. The supernatant was harvested and transferred to another Huh 7.5.1 cells. After $48 \mathrm{~h}$ incubation, the DENV and HCV-infected cells were determined by immunofluorescence assay. (B,C) Immunofluorescence assay, the green fluorescence showed the signals of DENV-2 NS3 protein and HCV core protein, DAPI indicated cell nuclei (left panels). The infectivity of mean $\pm S D$ was calculated from 3 observation fields (right panels). About 300 cells in each field were inspected. Data are mean $\pm S D$ from 3 independent tests. Statistical significance was tested by Student's $t$-test, ${ }^{\star} P<0.05,{ }^{\star \star \star} P<0.001$

resistance-associated variants are the negative predictors of sustained virological response (SVR; Manns et al., 2014). This finding has the implication that asunaprevir not only possesses a direct antiviral effect but also employs other mechanisms to restrict $\mathrm{HCV}$ replication, such that baseline resistance to asunaprevir did not affect the treatment outcome. The present study found that asunaprevir activated innate immunity and inhibited the replication of $\mathrm{HCV}$, which may enhance the direct anti-viral effect of asunaprevir in the treatment of HCV.

$\mathrm{HCV}$ directs a variety of strategies to disrupt host innate immune defenses through its viral proteins (Bartenschlager et al., 2012; Horner and Gale, 2013). HCV NS3-4A protease specifically cleaves both MAVS and TRIF to inactivate signals initiated by RIG-I and TLR3 and antagonizes innate immunity (Li K. et al., 2005; Li X. et al., 2005; Meylan et al., 2005; Liu and Gale, 2010). Therefore, it is anticipated that protease inhibitor can activate innate immunity upon inhibition of HCV NS3-4A protease in the context of HCV infection. However, interestingly, this study found that asunaprevir activated the MAVS mediated signaling pathway in Huh 7.5.1 cells not infected by HCV. After knockdown of MAVS but not TRIF with siRNA, the activation of MAVS mediated signaling pathway can be decreased. The present results demonstrated that asunaprevir activated the MAVS mediated signaling pathway in an HCV-independent but MAVS dependent manner. Moreover, after knockdown of MAVS but not TRIF with siRNA, the inhibition of asunaprevir on HCV replication can be partly rescued; evidencing that asunaprevir not only has direct anti-HCV effects but also activates innate immunity and restricts the replication of HCV.

The TLR-3 pathway is closely associated with the type I IFN signal pathway and after knock-down of MAVS, the type I IFN signal pathway also will be blocked (Bartenschlager et al., 2012; Horner and Gale, 2013). We found that asunaprevir treatment activated ISRE and IFN- $\beta$ promoter-luciferase activities and key signaling proteins in the type I IFN signal pathways in Huh 7.5.1 cells. In MAVS-knockdown cells, the restrictive effects of 


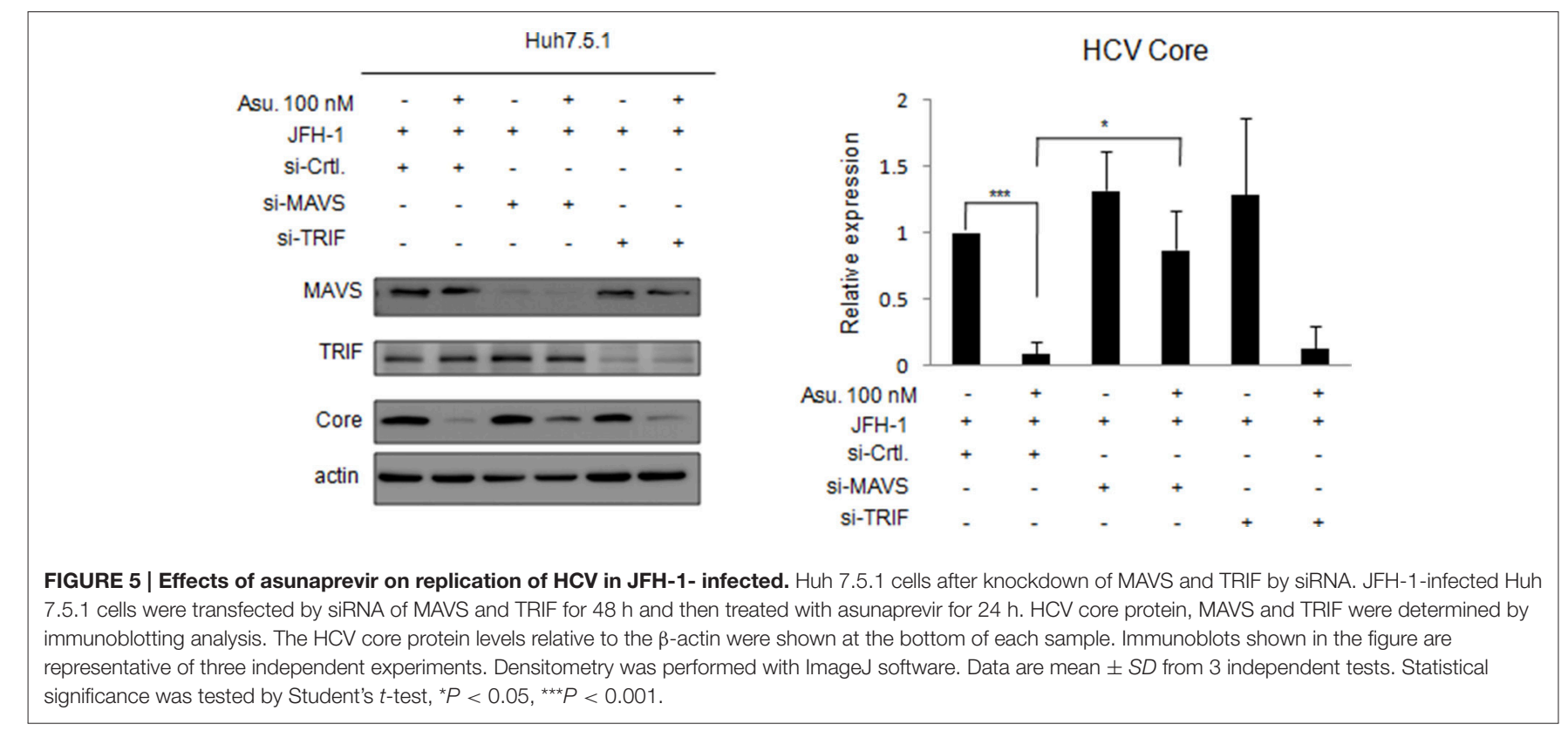

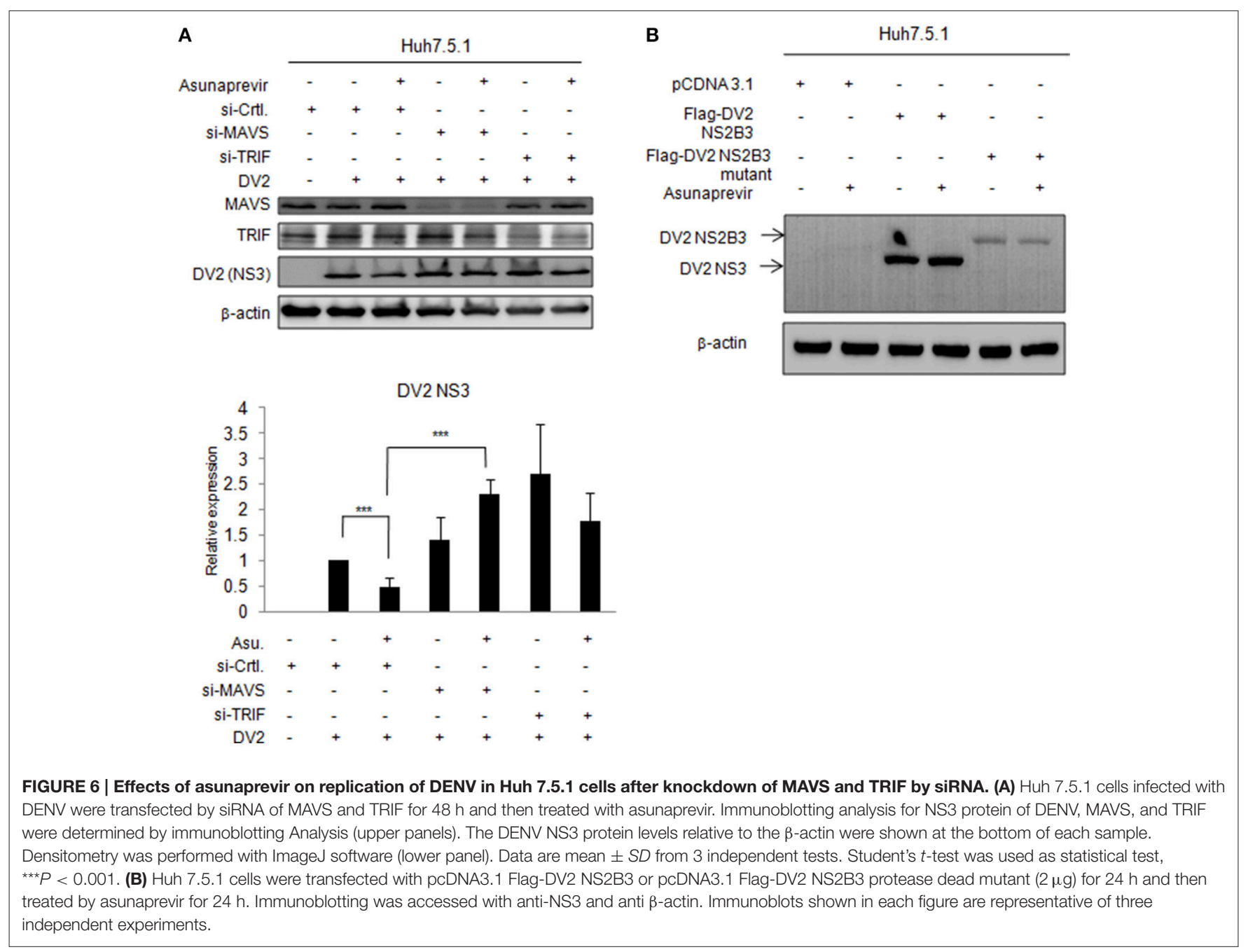


asunaprevir on HCV and DENV also were attenuated through the blockage of the anti-viral machinery.

Our results found that asunaprevir inhibited the replication of HCV and DENV. The influence of asunaprevir on the replication of HBV was performed, we found that asunaprevir also can inhibit the replication of HBV (Supplementary data Figure S1).We have also explored the effects of several other DAAs on ISRE luciferase activity and found that daclatasvir, MK5172 and sofosbuvir did not influence the ISRE luciferase activity (Supplementary data Figure S2).

Although Huh 7.5.1 cells were known not to express a functional RIG-I, but activation of MAVS independent of RIG-I is possible (Jacobs and Coyne, 2013). Our results found that asunaprevir increased MAVS expression and MAVS knockdown abolishes asunaprevir-induced MAVS mediated signaling pathway activation. This indicated that asunaprevir may activate the expression of MAVS independent of RIG-I.

Although DENV is an important global health issue, there has been no approved treatment for DENV. DENV can inhibit both type I IFN production and signaling in susceptible human cells. The NS2B3 protease complex of DENV acts an antagonist of type I IFN production. DENV also encodes proteins that antagonize type I IFN signaling, including NS2A, NS4A, NS4B, and NS5 by targeting different components of this signaling pathway, such as STATs. Importantly, the ability of the NS5 protein to bind and degrade STAT2 contributes to the limited host tropism of DENV to humans (Morrison et al., 2012; Green et al., 2014).

Treatment modality that triggers the host defense machinery by innate immune activation can restrict the replication of DENV (Olagnier et al., 2014; Wang et al., 2014) and may be an effective treatment option for DENV infection. Asunaprevir was designed to directly target protease of HCV to inhibit the

\section{REFERENCES}

Bartenschlager, R., Binder, M., and Thimme, R. (2012). Failure of innate and adaptive immune response in controlling hepatitis $\mathrm{C}$ virus infection. FEMS Microbiol. Rev. 36, 663-683. doi: 10.1111/j.1574-6976.2011. 00319.x

Chang, T. H., Kubota, T., Matsuoka, M., Jones, S., Bradfute, S. B., Bray, M., et al. (2009). Ebola virus VP35 blocks type I interferon transcription in dendritic cells by interacting with the cellular SUMOylation machinery. PLoS Pathog. 5:e1000493. doi: 10.1371/journal.ppat.1000493

Chang, T. H., Liao, C. L., and Lin, Y. L. (2006). Flavivirus induced interferonbeta gene expression through a pathway involving RIG-I-dependent IRF3 and PI3K-dependent NF-kappaB activation. Microb. Infect. 8, 157-171. doi: 10.1016/j.micinf.2005.06.014

Chayama, K., Takahashi, S., Toyota, J., Karino, Y., Ikeda, K., Ishikawa, H., et al. (2012). Dual therapy with the nonstructural protein 5A inhibitor, daclatasvir, and the nonstructural protein 3 protease inhibitor, asunaprevir, in hepatitis $\mathrm{C}$ virus genotype $1 \mathrm{~b}$-infected null responders. Hepatology 55, 742-748. doi: 10.1002/hep. 24724

Fattovich, G., Stroffolini, T., Zagni, I., and Donato, F. (2004). Hepatocellular carcinoma in cirrhosis: incidence and risk factors. Gastroenterology 127(Suppl. 1), S35-S50. doi: 10.1053/j.gastro.2004.09.014

Fusco, D. N., Brisac, C., John, S. P., Huang, Y. W., Chin, C. R., Xie, T., et al. (2013). A genetic screen identifies interferon- $\alpha$ effector genes required to suppress hepatitis C virus replication. Gastroenterology 144, 1438-1449. doi: 10.1053/j.gastro.2013.02.026 replication of HCV. Our data confirmed that DENV2 protease was not targeted by asunaprevir. Interestingly, asunaprevir was found to restrict also the replication of DENV in hepatoma cells. Similar to HCV inhibition, the inhibition of asunaprevir on DENV replication can be rescued in MAVS knockdown cells. Hence, asunaprevir restricts the replication of DENV by activating MAVS dependent innate immunity. Further, clinical study is needed to understand the effect of asunaprevir on DENV infection in clinical practice. In conclusion, asunaprevir activates innate immunity and restricts the replication of $\mathrm{HCV}$ and DENV through the activation of MAVS mediated innate immune response.

\section{AUTHOR CONTRIBUTIONS}

WT and TC designed the study. TC, WT, JC, HC, KL, CS, PH, and CW carried out data acquisition and analysis. WT and TC wrote the paper. RC and JC supervised the study. All authors reviewed the manuscript.

\section{FUNDING}

This study was supported by Ministry of Science and Technology of Taiwan, NSC- 102-2314-B-075B-002. Transparency declarations RC reports grants from Gilead, AbbVie, BristolMyers Squibb, Merck, Janssen and Mass Biologics. WT reports grants from Gilead.

\section{SUPPLEMENTARY MATERIAL}

The Supplementary Material for this article can be found online at: http://journal.frontiersin.org/article/10.3389/fmicb. 2017.00668/full\#supplementary-material

Green, A. M., Beatty, P. R., Hadjilaou, A., and Harris, E. (2012). Innate immunity evasion by Dengue virus. Viruses 4, 397-413. doi: 10.3390/v4030397

Green, A. M., Beatty, P. R., Hadjilaou, A., and Harris, E. (2014). Innate immunity to dengue virus infection and subversion of antiviral responses. J. Mol. Biol. 426, 1148-1160. doi: 10.1016/j.jmb.2013.11.023

Gubler, D. J. (1989). Aedes aegypti and Aedes aegypti-borne disease control in the 1990s: top down or bottom up. Charles Franklin Craig lecture. Am. J. Trop. Med. Hyg. 40, 571-578.

Horner, S. M., and Gale, M. Jr. (2013). Regulation of hepatic innate immunity by hepatitis C virus. Nat. Med. 19, 879-888. doi: 10.1038/nm.3253

Jacobs, J. L., and Coyne, C. B. (2013). Mechanisms of MAVS regulation at the mitochondrial membrane. J. Mol. Biol. 425, 5009-5019. doi: 10.1016/j.jmb. 2013.10.007

Kao, J. H., and Chen, D. S. (2005). Changing disease burden of hepatocellular carcinoma in the Far East and Souteast Asia. Liver Int. 25, 696-703. doi: 10.1111/j.1478-3231.2005.01139.x

Li, K., Foy, E., Ferreon, J. C., Nakamura, M., Ferreon, A. C., Ikeda, M., et al. (2005). Immune evasion by hepatitis C virus NS3/4A protease-mediated cleavage of the Toll-like receptor 3 adaptor protein TRIF. Proc. Natl. Acad. Sci. U.S.A. 102, 2992-2997. doi: 10.1073/pnas.0408824102

Li, K., Li, N. L., Wei, D., Pfeffer, S. R., Fan, M., and Pfeffer, L. M. (2012). Activation of chemokine and inflammatory cytokine response in hepatitis $\mathrm{C}$ virus-infected hepatocytes depends on Toll-like receptor 3 sensing of hepatitis $C$ virus doublestranded RNA intermediates. Hepatology 55, 666-675. doi: 10.1002/hep.24763

Li, X. D., Sun, L., Seth, R. B., Pineda, G., and Chen, Z. J. (2005). Hepatitis C virus protease NS3/4A cleaves mitochondrial antiviral signaling protein off 
the mitochondria to evade innate immunity. Proc. Natl. Acad. Sci. U.S.A. 102, 17717-17722. doi: 10.1073/pnas.0508531102

Liang, T. J., and Ghany, M. G. (2014). Therapy of hepatitis C-back to the future. N. Engl. J. Med. 370, 2043-2047. doi: 10.1056/NEJMe1403619

Lin, W., Tsai, W. L., Shao, R. X., Wu, G., Peng, L. F., Barlow, L. L., et al. (2010). Hepatitis C virus regulates transforming growth factor betal production through the generation of reactive oxygen species in a nuclear factor kappaB-dependent manner. Gastroenterology 138, 2509-2518. doi: 10.1053/j.gastro.2010.03.008

Lin, Y. L., Liao, C. L., Chen, L. K., Yeh, C. T., Liu, C. I., Ma, S. H., et al. (1998). Study of Dengue virus infection in SCID mice engrafted with human K562 cells. J. Virol. 2, 9729-9737.

Liu, H. M., and Gale, M. J. (2010). Hepatitis C virus evasion from RIGI-dependent hepatic innate immunity. Gastroenterol. Res. Pract. 8, 1-8. doi: $10.1155 / 2010 / 548390$

Manns, M., Pol, S., Jacobson, I. M., Marcellin, P., Gordon, S. C., Peng, C. Y., et al. (2014). All-oral daclatasvir plus asunaprevir for hepatitis $C$ virus genotype 1b: a multinational, phase 3, multicohort study. Lancet 384, 1597-1605. doi: 10.1016/S0140-6736(14)61059-X

Meylan, E., Curran, J., Hofmann, K., Moradpour, D., Binder, M., Bartenschlager, R., et al. (2005). Cardif is an adaptor protein in the RIG-I antiviral pathway and is targeted by hepatitis C virus. Nature 437, 1167-1172. doi: 10.1038 /nature04193

Morrison, J., Aguirre, S., and Fernandez-Sesma, A. (2012). Innate immunity evasion by Dengue virus. Viruses 4, 397-413. doi: 10.3390/v4030397

Noble, C. G., Chen, Y. L., Dong, H., Gu, F., Lim, S. P., Schul, W., et al. (2010). Strategies for development of Dengue virus inhibitors. Antiviral Res. 85, 450-462. doi: 10.1016/j.antiviral.2009.12.011

Olagnier, D., Scholte, F. E., Chiang, C., Albulescu, I. C., Nichols, C., He, Z., et al. (2014). Inhibition of Dengue and chikungunya virus infections by RIG-I-mediated type I interferon-independent stimulation of the innate antiviral response. J. Virol. 88, 4180-4194. doi: 10.1128/JVI. 03114-13

Ploss, A., and Dubuisson, J. (2012). New advances in the molecular biology of hepatitis $\mathrm{C}$ virus infection: towards the identification of new treatment targets. Gut 61(Suppl. 1), i25-i35. doi: 10.1136/gutjnl-2012-302048

Poole, R. M. (2014). Daclatasvir + asunaprevir: first global approval. Drugs 74, 1559-1571. doi: 10.1007/s40265-014-0279-4

Sameul, C. E. (2001). Antiviral actions of interferons. Clin. Microbiol. Rev. 14, 778-809. doi: 10.1128/CMR.14.4.778-809.2001
Schaefer, E. A., and Chung, R. T. (2012). Anti-hepatitis C virus drugs in development. Gastroenterology 142, 1340-1350.e1. doi: $10.1053 /$ j.gastro.2012.02.015

Schindler, C., and Plumlee, C. (2008). Interferons and the JAK-STAT pathway. Semin. Cell Dev. Biol. 19, 311-318. doi: 10.1016/j.semcdb.2008.08.010

Schoggins, J. W., and Rice, C. M. (2011). Interferon-stimulated genes and their antiviral effector functions. Curr. Opin. Virol. 1, 519-525. doi: 10.1016/j.coviro.2011.10.008

Schoggins, J. W., Wilson, S. J., Panis, M., Murphy, M. Y., Jones, C. T., Bieniasz, P., et al. (2011). A diverse range of gene products are effectors of the type I interferon antiviral response. Nature 472, 481-485. doi: 10.1038/nature09907

Tai, W. A., and Chung, R. T. (2009). Treatment failure in hepatitis C: mechanisms of non-response. J. Hepatol. 50, 412-420. doi: 10.1016/j.jhep.2008.11.010

Vaughn, D. W., Green, S., Kalayanarooj, S., Innis, B. L., Nimmannitya, S., Suntayakorn, S., et al. (2000). Dengue viremia titer, antibody response pattern, and virus serotype correlate with disease severity. J. Infect. Dis. 181, 2-9. doi: $10.1086 / 315215$

Wang, L. F., Lin, Y. S., Huang, N. C., Yu, C. Y., Tsai, W. L., Chen, J. J., et al. (2014). Hydroxychloroquine-Inhibited Dengue Virus Is associated with host defense machinery. J. Interferon Cytokine Res. 35, 143-156. doi: 10.1089/jir.2014.0038

Wang, N., Liang, Y., Devaraj, S., Wang, J., Lemon, S. M., and Li, K. (2009). Toll-like receptor 3 mediates establishment of an antiviral state against hepatitis $\mathrm{C}$ virus in hepatoma cells. J. Virol. 83, 9824-9834. doi: 10.1128/JVI.01125-09

WHO (2009). World Health Organization Geneva Switzerland and World Health Organization Dengue: Guidelines for Diagnosis, Treatment, Prevention, and Control. Geneva: World Health Organization.

WHO (2012). World Health Organization Geneva Switzerland and World Health Organization Dengue and Severe Dengue. Geneva: World Health Organization.

Conflict of Interest Statement: The authors declare that the research was conducted in the absence of any commercial or financial relationships that could be construed as a potential conflict of interest.

Copyright (C) 2017 Tsai, Cheng, Shu, Lai, Chan, Wu, Wu, Hsu, Chung and Chang. This is an open-access article distributed under the terms of the Creative Commons Attribution License (CC BY). The use, distribution or reproduction in other forums is permitted, provided the original author(s) or licensor are credited and that the original publication in this journal is cited, in accordance with accepted academic practice. No use, distribution or reproduction is permitted which does not comply with these terms. 